\title{
Gender Differences in the Effects of Cortisol and Dehydroepiandrosterone on Brain Derived Neurotrophic Factor in Patients with Major Depression
}

\author{
Hiroko SHUKUZAWA*1) 2), HaJIme BABA*1) 2), Hitoshi MAESHIMA*1) 2), TAKAHISA SHIMANO*2), \\ MEgumi INOUE*2), TAKao SAIDA*1) 2), ShUntaro NATSUME*1) 2), TOSHIHITO SUZUKI*1) 2) \\ *1) Department of Psychiatry \& Behavioral Science, Juntendo Graduate School of Medicine, Tokyo, Japan, *2) Juntendo \\ University Mood Disorder Project (JUMP), Department of Psychiatry, Juntendo Koshigaya Hospital, Saitama, Japan
}

Levels of serum brain derived neurotrophic factor (BDNF) are lower in patients with major depressive disorder (MDD). The adrenal cortex secretes the corticosteroids cortisol and dehydroepiandrosterone (DHEA) under stress condition, and serum levels of DHEA are gender-dependent. Although BDNF expression is associated with these corticosteroids, the effect of gender on this association is not clear. To examine gender differences in the relationship between BDNF and corticosteroids in patients with MDD, we measured serum levels of cortisol, DHEA-sulfate (DHEA-S) and BDNF in men and women with depression. We recruited 189 inpatients with MDD (76 men and 113 women) from Juntendo Koshigaya Hospital. Serum cortisol, DHEA-S and BDNF levels were measured on the morning after admission. Multiple regression analyses were conducted to assess the effects of cortisol and DHEA-S on BDNF. After controlling for age, we found that serum BDNF levels were significantly influenced by the DHEA-S/cortisol ratio $(p=0.010)$ in female participants. However, this association was absent in male participants. These results suggest gender differences exist in the effects of corticosteroids on BDNF. In female participants, the balance between neurotoxicity induced by cortisol and neuroprotection associated with DHEA-S may influence the pathology and symptoms of depression via BDNF.

Key words: brain derived neurotrophic factor (BDNF), dehydroepiandrosterone (DHEA), cortisol, depression, gender

\section{Introduction}

Major depressive disorder (MDD) is a leading cause of poor health and disability worldwide. More than 300 million people worldwide have MDD, and the number of patients is increasing ${ }^{1}$. However, the biological etiology and pathophysiology of MDD have not been fully elucidated.

Various studies have reported an association between levels of brain derived neurotrophic factor

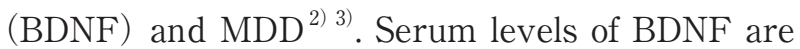
low in antidepressant-naïve patients with MDD in the depressive state ${ }^{4)}$ and increase with successful antidepressant treatment ${ }^{5}$. Negative correlations between serum levels of BDNF and depressive severity has been demonstrated ${ }^{6)}$. BDNF is a neurotrophin that influences neural development ${ }^{7{ }^{8}}$. It is expressed in various brain regions including the cortex and hippocampus and exerts beneficial effects on the development, survival and maintenance of neurons in the central nervous system ${ }^{9}$. BDNF production has been suggested to be modulated by corticosteroids such as cortisol and dehydroepiandrosterone (DHEA) ${ }^{10)}$.

Under stressful conditions, the neuroendocrine system is activated to facilitate self-defense, which is controlled by the hypothalamic-pituitary-adrenal axis (HPA axis). The HPA axis regulates

Corresponding author: Hajime Baba (ORCID iD: https://orcid.org/0000-0001-9678-8776)

Department of Psychiatry, Juntendo Koshigaya Hospital

560 Fukuroyama, Koshigaya-shi, Saitama 343-0032, Japan

TEL: + 81-48-975-0321 FAX: +81-48-975-3022 E-mail: hbaba@juntendo.ac.jp

〔Received Feb. 22, 2020〕〔Accepted Mar. 11, 2020]

J-STAGE Advance published date: May 15, 2020

Copyright (C) 2020 The Juntendo Medical Society. This is an open access article distributed under the terms of Creative Commons Attribution License (CC BY), which permits unrestricted use, distribution, and reproduction in any medium, provided the original source is properly credited. doi: 10.14789/jmj.2020.66.JMJ20-OA02 
glucocorticoid (mainly cortisol in humans) secretion by stimulating forward and feedback inhibition loops involving the brain, pituitary gland and adrenal gland ${ }^{11)}$. Patients with MDD exhibit hyperactivity in the HPA axis and increased cortisol secretion from the adrenal cortex ${ }^{12}$. Furthermore, both glucocorticoids and chronic stress reduce mRNA levels of $\mathrm{BDNF}^{10)}{ }^{13)-15}$ )

DHEA and its sulfate (DHEA-S) have also been implicated in the pathophysiology of $\mathrm{MDD}^{10)}{ }^{16)}$. Although the data are inconsistent, most studies have demonstrated that depressed individuals exhibit lower plasma/serum levels of DHEA and DHEA-S and have reported a negative correlation between DHEA-S levels and depressive symptoms ${ }^{16)}$. A recent meta-analysis also showed lower serum DHEA-S levels in patients with depression compared with healthy controls ${ }^{17}$. DHEA and DHEA-S are secreted from the adrenal cortex and testis. They are precursors to sex hormones, that is, they are transformed to androgens and estrogens in peripheral tissues and they are the most abundant steroid hormones in the human body ${ }^{18)}{ }^{19}$. Previous studies have indicated that DHEA and DHEA-S may modulate BDNF production ${ }^{16)}$. For instance, DHEA induces BDNF overproduction in the cerebral cortex ${ }^{20)}$. Furthermore, DEHA and DHEA-S have different modulatory effects on BDNF production in rats. Specifically, DHEA increases BDNF in the hypothalamus and DHEA-S increases BDNF in the hippocampus ${ }^{21)}$. Serum levels of DHEA-S are 250 and 500 times higher than those of DHEA in men and women, respectively ${ }^{19}$, and men have higher levels of DHEA-S than women ${ }^{22-25)}$. Moreover, although the mechanism remains unclear, DHEA and DHEA-S are thought to have an anti-glucocorticoid effect ${ }^{16)}{ }^{26)}$. As these steroids co-regulate one another, it is important to consider the DHEA-S/ cortisol ratio in addition to their absolute levels ${ }^{16}$.

Given the gender differences in DHEA-S production, we hypothesized that the relationship between BDNF and corticosteroids may differ between men and women with depression. In the present study, we analyzed the relationships among serum levels of BDNF, cortisol and DHEA-S, as well as the DHEA-S/cortisol ratio in men and women with MDD.

\section{Methods}

\section{Participants}

In this cross-sectional study, 192 inpatients who met the criteria of the Diagnostic and Statistical Manual for Mental Disorders, 4th edition (DSMIV) for MDD were recruited from the Juntendo Koshigaya Hospital between April 2005 and July 2011. Eight senior psychiatrists made the diagnoses. Exclusion criteria included a history of other psychiatric disorders including delusions, severe or acute medical illnesses (such as cancer, severe inflammatory disease, severe heart disease, severe diabetes or severe hormonal disease), neurological disorders, use of drugs that have been found to cause depression (such as interferons, antiestrogens or steroids), addiction, clinical evidence of dementia and Mini-Mental State Examination scores <24. Finally, we enrolled 189 participants with MDD (76 men, 113 women; mean age 54.7 years; range, $26-79$ years). Depressive symptoms were assessed using the Hamilton Rating Scale for Depression (HAM-D) ${ }^{27)}$ upon admission to the hospital. The number of depressive episodes and total duration of illness were confirmed via medical records. All participants were currently receiving antidepressant medication. Sixty-seven participants were taking tricyclic antidepressants (TCA), 68 were taking non-TCA antidepressants (tetracyclic antidepressants, trazodone), 42 were taking selective serotonin reuptake inhibitors (SSRI), 30 were taking serotonin noradrenalin reuptake inhibitors (SNRI) and 13 were taking mirtazapine. Thirtyone participants were taking two different antidepressants. The antidepressant doses were converted to equivalent doses of imipramine ${ }^{28}$.

The study protocol was approved by the Medical Ethics Committee of Juntendo University. The study was performed in accordance with the regulations outlined by Juntendo University and conformed to the provisions of the Declaration of Helsinki (1995). All participants provided written informed consent prior to participation.

\section{Experimental procedure}

Blood samples were taken before breakfast at 07:00 on the day after admission and were centrifuged immediately after blood was drawn and clotting confirmed. Serum samples were stored at 
$-80^{\circ} \mathrm{C}$ until the time of processing.

Serum levels of BDNF were measured using a Quantikine Human BDNF Immunoassay Kit (R\&D Systems, Minneapolis, MN, USA) according to the instructions from the manufacturer and as previously described ${ }^{29)}$. The lower limit of the detection was $20 \mathrm{pg} / \mathrm{ml}$ and the intra-assay coefficient of variation was $<8 \%$.

Serum levels of cortisol were measured via competitive immunoassay using the ADVIA Centaur Cortisol assay (Siemens Healthcare Diagnostic Inc., Tokyo, Japan). This assay measures serum cortisol concentrations up to $75 \mu \mathrm{g} / \mathrm{d} l(2,069 \mathrm{nmol} / l)$ with a minimum detectable concentration (analytical sensitivity) of $0.20 \mu \mathrm{g} / \mathrm{d} l(5.5 \mathrm{nmol} / l)$. The intra-assay CVs were $<15 \%{ }^{30}$.

Serum levels of DHEA-S were measured at the SRL Laboratory (Tokyo, Japan) using a chemiluminescent immunoassay (CLEIA; Access DHEA-S, Beckman Coulter KK, Tokyo, Japan). The lower limit of the detection for DHEA-S were $11 \mathrm{ng} / \mathrm{m} l$. Inter- and intra-assay coefficients of variation were $10.6 \%$ and $7.2 \%$ for DHEA-S ${ }^{25)}$.

\section{Statistical analysis}

For statistical analysis, we compared age, HAM-D score, number of depressive episodes, total duration of medication, total dose of antidepressants (converted to equivalent doses of imipramine) and MMSE score, as well as serum levels of BDNF, cortisol, DHEA-S and the DHEA-S/cortisol ratio between male and female participants using a twotailed unpaired Student's t-test or Mann-Whitney U test. Correlations between HAM-D score or BDNF levels and corticosteroids were analyzed using Spearman's rank correlation coefficient test. Multiple regression analyses were conducted using serum levels of BDNF as the dependent variable, and age, serum levels of DHEA-S, serum levels of cortisol and the DHEA-S/cortisol ratio as independent variables. To analyze the effects of cortisol and DHEA-S on BDNF by gender, we used a significance level of $p<0.025$ with Bonferroni correction. Statistical procedures were performed using the Japanese version of SPSS v21.0 (SPSS Japan, Tokyo, Japan).

\section{Results}

The detailed demographic data and serum levels of BDNF, cortisol, DHEA-S and the DHEA-S/cortisol ratio at admission by gender are shown in Table -1 . The mean age $(p=0.004)$ and HAM-D score $(p=0.021)$ were significantly higher in female than male participants. Although the Kolmogorov-Smirnov test confirmed normal distribution for the BDNF $(p=0.20)$ and cortisol $(p=0.07)$ data, the DHEA-S data had a skewed distribution $(\mathrm{p}<0.001)$. Therefore, we used Student's t-tests to compare the BDNF and cortisol data and the MannWhitney $\mathrm{U}$ test to compare the DHEA-S and

Table-1 Demographic data and serum levels of BDNF, cortisol and DHEA-S in participants with MDD

\begin{tabular}{|c|c|c|c|}
\hline & Male $(\mathrm{n}=76)$ & Female $(\mathrm{n}=113)$ & \multirow{2}{*}{$\mathrm{p}$ value } \\
\hline & Mean (SD) & Mean (SD) & \\
\hline Age (Years) & $51.0(13.0)$ & $57.1(14.2)$ & $0.004^{\mathrm{a}}$ \\
\hline Ham-D score & $18.9(9.6)$ & $23.1(10.1)$ & $0.021^{\mathrm{a}}$ \\
\hline Number of Depressive Episodes & $2.1(1.7)$ & $2.1(1.3)$ & $0.913^{\mathrm{a}}$ \\
\hline Total Duration of Medication (months) & $21.9(26.9)$ & $31.9(60.9)$ & $0.838^{\mathrm{a}}$ \\
\hline Total Dose of Antidepressant $(\mathrm{mg})^{\mathrm{c}}$ & $144.2(81.5)$ & $119.4(77.6)$ & $0.077^{\mathrm{a}}$ \\
\hline MMSE score & $26.8(2.3)$ & $26.6(2.8)$ & $0.798^{\mathrm{a}}$ \\
\hline BDNF $(\mathrm{pg} / \mathrm{m} l)$ & $21,822.5(7,843.6)$ & $20,130.6(6,228.6)$ & $0.101^{\mathrm{a}}$ \\
\hline Cortisol $(\mu \mathrm{g} / \mathrm{d} l)$ & $16.1(6.1)$ & $15.8(6.7)$ & $0.772^{\mathrm{a}}$ \\
\hline DHEA-S $(\mathrm{ng} / \mathrm{m} l)$ & $878.6(952.0)$ & $489.1(636.8)$ & $<0.001^{\mathrm{b}}$ \\
\hline DHEA-S/cortisol & $70.6(108.4)$ & $39.7(65.3)$ & $0.001^{\mathrm{b}}$ \\
\hline
\end{tabular}

a: Student-T-test, b: Mann-Whitney U test, c: Antidepressant doses were converted into imipramine equivalent doses.

MDD: Major Depressive Disorder, HAM-D: Hamilton rating scale of depression,

MMSE: Mini-Mental State Examination, 
DHEA-S/cortisol data. Serum levels of BDNF and cortisol did not differ between male and female participants. Serum levels of DHEA-S $(p<0.001)$ and the DHEA-S/cortisol ratio $(\mathrm{p}=0.001)$ were significantly higher in male than female participants.

In terms of the relationship between the severity of depressive symptoms and levels of corticosteroids, we found that the total HAM-D score was negatively correlated with serum levels of DHEA-S $(\mathrm{R}=-0.308, \mathrm{p}=0.022)$ and the DHEA-S/cortisol ratio $(\mathrm{R}=-0.332, \mathrm{p}=0.013)$ in male participants, while this correlation was not significant in female participants. Conversely, while serum levels of BDNF were positively correlated with those of DHEA-S $(\mathrm{R}=314, \mathrm{p}=0.001)$ and the DHEA-S/ cortisol ratio $(\mathrm{R}=0.332, \mathrm{p}<0.001)$ in female participants, these correlations were not significant in male participants (Table-2).

The DHEA-S and DHEA-S/cortisol data were $\log 10$ transformed prior to multiple regression analysis because the DHEA-S data had a skewed distribution. The results of the multiple regression analysis indicated that age affected BDNF levels in both male and female participants. After controlling for age, we found no effect of cortisol levels,
DHEA-S levels, or the DHEA-S/cortisol ratio on BDNF levels in male participants with MDD. In female participants, serum levels of BDNF were not strongly $(\beta=0.224)$ but significantly influenced by the DHEA-S/cortisol ratio $(\mathrm{p}=0.019)$. Furthermore, DHEA-S tended to have a positive effect on BDNF levels $(p=0.026$; Table-3).

\section{Discussion}

The main finding of the present study was the presence of gender differences in the relationship between serum levels of BDNF and corticosteroids in participants with MDD. While serum levels of BDNF appeared to be associated with the DHEA-S/cortisol ratio in female participants with MDD, this association was absent in male participants.

As mentioned above, patients with depression exhibit increased serum levels of cortisol as the result of hyperactivity in the HPA axis ${ }^{12}$. Furthermore, studies have demonstrated decreased serum levels of DHEA-S in depressed individuals ${ }^{16}$. BDNF expression and production decreased with heightened cortisol levels and to increase after the application of DHEA ${ }^{10)}$ ) . Although the mechanisms

Table-2 Correlations between HAM-D score or BDNF and corticosteroids ${ }^{\mathrm{a}}$

\begin{tabular}{|c|c|c|c|c|c|c|c|c|}
\hline & \multicolumn{4}{|c|}{ HAM-D score } & \multicolumn{4}{|c|}{$\mathrm{BDNF}$} \\
\hline & \multicolumn{2}{|c|}{ Male } & \multicolumn{2}{|c|}{ Female } & \multicolumn{2}{|c|}{ Male } & \multicolumn{2}{|c|}{ Female } \\
\hline & $\mathrm{R}$ & $\mathrm{p}$ value & $\mathrm{R}$ & $\mathrm{p}$ value & $\mathrm{R}$ & $\mathrm{p}$ value & $\mathrm{R}$ & $\mathrm{p}$ value \\
\hline Cortisol $(\mu \mathrm{g} / \mathrm{d} l)$ & 0.045 & 0.744 & 0.063 & 0.603 & 0.078 & 0.501 & -0.119 & 0.211 \\
\hline DHEA-S $(\mathrm{ng} / \mathrm{m} l)$ & -0.308 & 0.022 & -0.237 & 0.046 & 0.243 & 0.034 & 0.314 & 0.001 \\
\hline DHEA-S/cortisol & -0.332 & 0.013 & -0.215 & 0.072 & 0.199 & 0.085 & 0.332 & $<0.001$ \\
\hline
\end{tabular}

a: Spearman's rank correlation coefficient

HAM-D: Hamilton rating scale of depression

Table-3 Multiple regression analysis of BDNF levels

\begin{tabular}{|c|c|c|c|c|c|c|c|c|}
\hline & \multicolumn{8}{|c|}{ BDNF } \\
\hline & \multicolumn{4}{|c|}{ Male } & \multicolumn{4}{|c|}{ Female } \\
\hline & \multicolumn{2}{|c|}{ Model 1} & \multicolumn{2}{|c|}{ Model 2} & \multicolumn{2}{|c|}{ Model 1} & \multicolumn{2}{|c|}{ Model 2} \\
\hline & $\beta$ Estimate & $\mathrm{p}$ value & $\beta$ Estimate & $\mathrm{p}$ value & $\beta$ Estimate & $\mathrm{p}$ value & $\beta$ Estimate & $\mathrm{p}$ value \\
\hline Age & -0.328 & 0.007 & -0.326 & 0.008 & -0.241 & 0.012 & -0.236 & 0.014 \\
\hline Cortisol & -0.078 & 0.490 & - & - & -0.029 & 0.749 & - & - \\
\hline $\log _{10}$ DHEA-S & 0.113 & 0.337 & - & - & 0.213 & 0.026 & - & - \\
\hline $\log _{10}$ DHEA-S/Cortisol & - & - & 0.075 & 0.527 & - & - & 0.224 & 0.019 \\
\hline
\end{tabular}

Model 1; input age, cortisol and DHEA-S as independent variables

Model 2; input age and DHEA-S/cortisol ratio as independent variables 
underlying the gender differences found in the relationship between serum levels of BDNF and the DHEA-S/cortisol ratio in the present study are unclear, previous studies have indicated that there may be gender differences in BDNF production under stressful conditions or in depressed individuals. Kreinin et $a l^{31)}$ found a positive correlation between BDNF levels and depression severity among untreated female patients with MDD. Furthermore, when Ambrus et al ${ }^{32)}$ investigated the relationship between HPA axis activity and BDNF levels in suicide attempters using the dexamethasone suppression test (DST), they found that hyperactivity in the HPA axis was associated with decreased BDNF plasma levels only in female participants. Furthermore, animal studies have suggested sex differences in how stress modulates BDNF expression ${ }^{33}$. These findings indicate that the relationship between BDNF levels and depressive symptoms may be stronger in women than men.

Consistent with previous reports, we observed higher serum levels of DHEA-S in male versus female participants in the present study. This is consistent with previous studies that reported gender differences in DHEA-S levels in patients with depression. For instance, Goldman and Glei ${ }^{24}$ reported a negative correlation between serum levels of DHEA-S and depressive symptoms in male patients, but not in female patients. Furthermore, Michikawa et al. ${ }^{34)}$ investigated baseline serum levels of DHEA-S in older subjects without depressive symptoms and conducted follow-up assessments to evaluate the incidence of depressive symptoms. They found that a high level of serum DHEA-S was associated with a reduced risk of depressive symptoms in men but not in women, which suggests that DHEA-S may have a protective effect against the development of depressive symptoms in men but not in women. Finally, we previously investigated serum levels of DHEA-S in participants with MDD and healthy controls, and found that serum levels of DHEA-S in male patients were significantly decreased compared with male controls, whereas no significant differences were seen between female patients and female controls ${ }^{25}$. The results of the correlation analysis in the present study indicate that serum levels of DHEA-S were negatively correlated with depressive symptoms in male participants and positively correlated with serum BDNF levels in female participants. Considering our present data together with previous reports, it is possible to speculate that depression in male patients may be directly affected by a decreased neuroprotective effect of DHEA-S, while a decline in DHEA-S levels may influence depression in female patients via decreased BDNF levels.

There are several limitations to the present study. First, all participants in this study were receiving antidepressant medication. Antidepressants alter serum BDNF levels and DHEA-S levels in participants with MDD. With respect to the relationship between antidepressant treatment and BDNF levels, serum levels of BDNF increase only in patients who respond to treatment, even among those who are treated with same antidepressant ${ }^{35)}{ }^{36)}$. Similarly, remitted and non-remitted patients who received antidepressants exhibited different levels of change in terms of serum levels of DHEA and DHEA-S ${ }^{37}$. These reports indicate that serum levels of BDNF and DHEA-S may not be directly affected by antidepressants. However, to avoid the influence of antidepressants completely, further studies should examine drug-naive patients. Second, the female participants in this study varied widely in terms of age, such that the group included pre- $^{-}$and post-menopausal women. We reanalyzed the data after dividing the participants into two age groups (younger: $<60$ years, $\mathrm{n}=104$, and older: $\geq$ 60 years, $\mathrm{n}=85$ ). While we found similar tendencies for all participants (when grouped by gender) in the younger group, these tendencies were not observed in the older group (data not shown). Age and menstrual cycle affect activity in the HPA axis ${ }^{38}{ }^{39)}$. Although postmenopausal older individuals $^{40)}$ and perimenopausal individuals ${ }^{41)}$ exhibit similar decreases in serum levels of DHEA-S, further studies that control for these confounding factors are necessary. Third, all participants were inpatients at the same hospital. Therefore, the lighting schedule, wake-up (temperature measurement) time and mealtimes were consistent. However, the sleep and eating conditions were not controlled in this study. Because sleep and eating conditions can influence mental state, BDNF levels and corticosteroid levels, these confounding factors represent limitations of the present study. Finally, although they were statistically significant, 
the correlation coefficients in the correlation analysis and the $\beta$ estimate in the multiple regression analysis were relatively small. From a clinical perspective, this may have affected the study impact. However, our observations regarding possible gender differences in the relationship between corticosteroids and BDNF levels may be an important jumping-off point for further studies concerning gender and depression.

In conclusion, we observed gender differences in the relationship between BDNF and corticosteroids (cortisol and DHEA-S) in participants with depression. Serum levels of BDNF appeared to be associated with the DHEA-S/cortisol ratio in female participants only, and the levels of DHEA-S appeared to be directly correlated with depression severity in male participants. This suggests that depression may be directly related to a decreased neuroprotective effect of DHEA-S in men, while an altered corticosteroid balance involving decreased DHEA-S levels may influence depression via decreased BDNF levels in women. These results suggest that the etiology of depression may have gender differences.

\section{Acknowledgments}

This work was supported by grants from the Research Support Foundation of the Juntendo Institute of Mental Health.

\section{Conflicts of interest}

No disclosures to report.

\section{References}

1) WHO, 2017.

2) Kishi T, Yoshimura R, Ikuta T, Iwata N: Brain-Derived Neurotrophic Factor and Major Depressive Disorder: Evidence from Meta-Analyses. Front Psychiatry, 2018; 8: 308.

3) Polyakova M, Stuke K, Schuemberg K, Mueller K, Schoenknecht P, Schroeter ML: BDNF as a biomarker for successful treatment of mood disorders: a systematic \& quantitative meta-analysis. J Affect Disord, 2015; 174: 432-440.

4) Kang HJ, Kim JM, Lee JY, et al: BDNF promoter methylation and suicidal behavior in depressive patients. J Affect Disord, 2013; 151: 679-685.

5) Martinotti G, Pettorruso M, De Berardis D, et al: Agomelatine Increases BDNF Serum Levels in Depressed Patients in Correlation with the Improvement of Depressive Symptoms. Int J Neuropsychopharmacol, 2016; 19. pii: pyw003.

6) Satomura E, Baba H, Nakano Y, Maeshima H, Suzuki T,
Arai H: Correlations between brain-derived neurotrophic factor and clinical symptoms in medicated patients with major depression. J Affect Disord, 2011; 135: 332335.

7) Berkemeier LR, Winslow JW, Kaplan DR, Nikolics K, Goeddel DV, Rosenthal A: Neurotrophin-5: a novel neurotrophic factor that activates trk and trkB. Neuron, 1991; 7: 857-866.

8) Noureddini M, Verdi J, Mortazavi-Tabatabaei SA, et al: Human endometrial stem cell neurogenesis in response to NGF and bFGF. Cell Biol Int, 2012; 36: 961-966.

9) Numakawa T, Odaka H, Adachi N: Actions of BrainDerived Neurotrophin Factor in the Neurogenesis and Neuronal Function, and Its Involvement in the Pathophysiology of Brain Diseases. Int J Mol Sci, 2018; 19. pii: E3650.

10) Pluchino N, Russo M, Santoro AN, Litta P, Cela V, Genazzani AR: Steroid hormones and BDNF. Neuroscience, 2013; 239: 271-279.

11) Keller J, Gomez R, Williams G, et al: HPA axis in major depression: cortisol, clinical symptomatology and genetic variation predict cognition. Mol Psychiatry, 2017; 22: 527-536.

12) Pariante CM, Lightman SL: The HPA axis in major depression: classical theories and new developments. Trends Neurosci, 2008; 31: 464-468.

13) Smith MA, Makino S, Kvetnansky R, Post RM: Stress and glucocorticoids affect the expression of brainderived neurotrophic factor and neurotrophin-3 mRNAs in the hippocampus. J Neurosci, 1995; 15 (3 Pt 1): 17681777.

14) Schaaf MJ, de Jong J, de Kloet ER, Vreugdenhil E: Downregulation of BDNF mRNA and protein in the rat hippocampus by corticosterone. Brain Res, 1998; 813: $112-120$

15) Rojas PS, Fritsch R, Rojas RA, Jara P, Fiedler JL: Serum brain-derived neurotrophic factor and glucocorticoid receptor levels in lymphocytes as markers of antidepressant response in major depressive patients: a pilot study. Psychiatry Res, 2011; 189: 239-245.

16) Maninger N, Wolkowitz OM, Reus VI, Epel ES, Mellon SH: Neurobiological and neuropsychiatric effects of dehydroepiandrosterone (DHEA) and DHEA sulfate (DHEAS). Front Neuroendocrinol, 2009; 30: 65-91.

17) Zhu G, Yin Y, Xiao CL, et al: Serum DHEAS levels are associated with the development of depression. Psychiatry Res, 2015; 229: 447-453.

18) Nieschlag E, Loriaux DL, Ruder HJ, Zucker IR, Kirschner MA, Lipsett MB: The secretion of dehydroepiandrosterone and dehydroepiandrosterone sulphate in man. J Endocrinol, 1973; 57: 123-134.

19) Labrie F, Bélanger A, Cusan L, Gomez JL, Candas B: Marked decline in serum concentrations of adrenal C19 sex steroid precursors and conjugated androgen metabolites during aging. J Clin Endocrinol Metab, 1997; 82: 2396-2402

20) Rahmani A, Shoae-Hassani A, Keyhanvar P, Kheradmand D, Darbandi-Azar A: Dehydroepiandrosterone stimulates nerve growth factor and brain derived neurotrophic factor in cortical neurons. Adv Pharmacol Sci, 2013; 2013: 506191.

21) Naert G, Maurice T, Tapia-Arancibia L, Givalois L: Neuroactive steroids modulate HPA axis activity and cerebral brain-derived neurotrophic factor (BDNF) protein levels in adult male rats. Psychoneuroendocrinology, 
2007; 32: 1062-1078.

22) Sulcová J, Hill M, Hampl R, Stárka L: Age and sex related differences in serum levels of unconjugated dehydroepiandrosterone and its sulphate in normal subjects. J Endocrinol, 1997; 154: 57-62.

23) Yamaji T, Ibayashi H: Plasma dehydroepiandrosterone sulfate in normal and pathological conditions. J Clin Endocrinol Metab, 1969; 29: 273-278.

24) Goldman N, Glei DA: Sex differences in the relationship between DHEAS and health. Exp Gerontol, 2007; 42: 979-987.

25) Kurita H, Maeshima H, Kida S, et al: Serum dehydroepiandrosterone (DHEA) and DHEA-sulfate (S) levels in medicated patients with major depressive disorder compared with controls. J Affect Disord, 2013; 146: 205212

26) Mocking RJ, Pellikaan CM, Lok A, et al: DHEAS and cortisol/DHEAS-ratio in recurrent depression: State, or trait predicting 10-year recurrence? Psychoneuroendocrinology, 2015; 59: 91-101.

27) Hamilton M: A rating scale for depression. J Neurol Neurosurg Psychiatry, 1960; 23: 56-62.

28) Inada T, Inagaki A: Psychotropic dose equivalence in Japan. Psychiatry Clin Neurosci, 2015; 69: 440-447.

29) Minelli A, Zanardini R, Bonvicini C, et al: BDNF serum levels, but not BDNF Val66Met genotype, are correlated with personality traits in healthy subjects. Eur Arch Psychiatry Clin Neurosci, 2011; 261: 323-329.

30) Ishijima S, Baba H, Maeshima H, et al: Glucocorticoid may influence amyloid $\beta$ metabolism in patients with depression. Psychiatry Res, 2018; 259: 191-196.

31) Kreinin A, Lisson S, Nesher E, et al: Blood BDNF level is gender specific in severe depression. PLoS One, 2015; 10: e0127643.

32) Ambrus L, Lindqvist D, Träskman-Bendz L, Westrin $\AA$ : Hypothalamic-pituitary-adrenal axis hyperactivity is associated with decreased brain-derived neurotrophic factor in female suicide attempters. Nord J Psychiatry, 2016; 70: 575-581.
33) Bath KG, Schilit A, Lee FS: Stress effects on BDNF expression: effects of age, sex, and form of stress. Neuroscience, 2013; 239: 149-156.

34) Michikawa T, Nishiwaki Y, Nakano M, et al: Higher serum dehydroepiandrosterone sulfate levels are protectively associated with depressive symptoms in men, but not in women: a community-based cohort study of older Japanese. Am J Geriatr Psychiatry, 2013; 21: 1154-1163.

35) Shimizu E, Hashimoto K, Okamura N, et al: Alterations of serum levels of brain-derived neurotrophic factor (BDNF) in depressed patients with or without antidepressants. Biol Psychiatry, 2003; 54: 70-75.

36) Yoshimura R, Mitoma M, Sugita A, et al: Effects of paroxetine or milnacipran on serum brain-derived neurotrophic factor in depressed patients. Prog Neuropsychopharmacol Biol Psychiatry, 2007; 31: 1034-1037.

37) Fabian TJ, Dew MA, Pollock BG, et al: Endogenous concentrations of DHEA and DHEA-S decrease with remission of depression in older adults. Biol Psychiatry, 2001; 50: 767-774.

38) Kirschbaum C, Kudielka BM, Gaab J, Schommer NC, Hellhammer DH: Impact of gender, menstrual cycle phase, and oral contraceptives on the activity of the hypothalamus-pituitary-adrenal axis. Psychosom Med, 1999; 61: 154-162.

39) Keenan DM, Roelfsema F, Carroll BJ, Iranmanesh A, Veldhuis JD: Sex defines the age dependence of endogenous ACTH-cortisol dose responsiveness. Am J Physiol Regul Integr Comp Physiol, 2009; 297: R515-523.

40) Barrett-Connor E, von Mühlen D, Laughlin GA, Kripke A: Endogenous levels of dehydroepiandrosterone sulfate, but not other sex hormones, are associated with depressed mood in older women: the Rancho Bernardo Study. J Am Geriatr Soc, 1999; 47: 685-691.

41) Schmidt PJ, Murphy JH, Haq N, Danaceau MA, St Clair $\mathrm{L}$ : Basal plasma hormone levels in depressed perimenopausal women. Psychoneuroendocrinology, 2002; 27: 907-920. 